\title{
New type of zero-field steps in the I-V curve of a Josephson junction of intermediate length
}

Hansen, Jørn Bindslev; Mygind, Jesper

\section{Published in:}

Physical Review B

Link to article, DOI:

10.1103/PhysRevB.32.178

Publication date:

1985

Document Version

Publisher's PDF, also known as Version of record

Link back to DTU Orbit

Citation (APA):

Hansen, J. B., \& Mygind, J. (1985). New type of zero-field steps in the I-V curve of a Josephson junction of intermediate length. Physical Review B, 32(1), 178-181. https://doi.org/10.1103/PhysRevB.32.178

\section{General rights}

Copyright and moral rights for the publications made accessible in the public portal are retained by the authors and/or other copyright owners and it is a condition of accessing publications that users recognise and abide by the legal requirements associated with these rights.

- Users may download and print one copy of any publication from the public portal for the purpose of private study or research.

- You may not further distribute the material or use it for any profit-making activity or commercial gain

- You may freely distribute the URL identifying the publication in the public portal 


\title{
New type of zero-field steps in the $I$ - $V$ curve of a Josephson junction of intermediate length
}

\author{
J. Bindslev Hansen \\ Physics Laboratory I, H. C. Ørsted Institute, University of Copenhagen, DK-2100 Copenhagen $\emptyset$, Denmark
}

\section{J. Mygind}

Physics Laboratory I, The Technical University of Denmark, DK-2800 Lyngby, Denmark

(Received 10 December 1984)

\begin{abstract}
We report on the observation of a new type of high-order zero-field steps in the $I-V$ curve of a Josephson transmission line of intermediate length $l=L / \lambda_{J} \approx 5$, where $\lambda_{J}$ is the Josephson penetration length. On such steps, deviations from the generalized Josephson relation $V_{\mathrm{dc}}=N(h / 2 e) v_{\mathrm{det}}$ relating the measured dc voltage to the frequency of the radiation emitted from the junction are observed.
\end{abstract}

\section{INTRODUCTION}

The Josephson transmission line constitutes a model system for the study of solitary waves and other excitations in $(s+t)=(1+1)$ dimensional, nonlinear systems described by the perturbed sine-Gordon equation. ${ }^{1}$ In a Josephson junction a soliton (or fluxon) is a localized $\pm 2 \pi$ "kink" in the phase difference across the tunnel barrier between two superconductors. The solitary wave character of this propagating phase kink has recently been confirmed by direct time-resolved measurements on long and narrow Josephson tunnel junctions. ${ }^{2}$ One of the manifestations of soliton excitations in such a structure is the appearance of zero-field current steps (ZFS) in the dc $I-V$ curve. $^{3}$ As an experimental "rule of thumb" the number of ZFS, regularly spaced along the voltage axis, is given by $L / \lambda_{J}$. The Josephson penetration length $\lambda_{J}$ measures the spatial extent of a stationary fluxon. The line may be considered one-dimensional when $l=L / \lambda_{J} \gg 1$ and $w=W / \lambda_{J} \ll 1$. Here $L$ and $W$ are the length and the width of the junction. In a magnetic field additional steps, Fiske steps (FS), appear. These steps may also be explained within the soliton picture, if the propagating fluxons are combined with plasmon or plasmonlike excitations on the line. ${ }^{4}$

\section{THEORY}

A one-dimensional Josephson tunnel junction is generally accepted to be described by the perturbed sineGordon equation, ${ }^{5}$ which without surface losses may be written as

$$
-\phi_{x x}+\phi_{t t}+\alpha \phi_{t}+\sin \phi=\eta,
$$

where the subscripts stand for partial derivatives. Here $\phi(x, t)$ is the space- and time-dependent phase difference between the two superconducting films. The time, $t$, is measured in units of the reciprocal maximum plasma frequency, $\omega_{0}^{-1}$, where $\omega_{0}=\bar{c} / \lambda_{J}=(2 e J / \hbar C)^{1 / 2}$. The maximum velocity, $\bar{c}$, is the propagation velocity of (Swihart mode) electromagnetic waves on the identical superconducting transmission line free from nonlinear effects caused by the $\sin \phi$ term. The spatial variable, $x$, is mea- sured in units of $\lambda_{J}=\left(\hbar / 2 \mu_{0} e d J\right)^{1 / 2} . J$ is the maximum pair current density and $d$ is the magnetic thickness of the barrier. The parameter $\alpha$ is the damping constant, $\alpha=G(\hbar / 2 e J C)^{1 / 2}$, where $C$ and $G$ are the shunt capacitance and conductance per unit area (the quasiparticle conductance), respectively. $\alpha=\beta_{c}^{-1 / 2}$, where $\beta_{c}$ is the McCumber parameter. For an overlap junction (see inset in Fig. 1) with uniform current distribution $\eta=I_{\mathrm{dc}} / I_{0}^{\mathrm{ov}}$. Here $I_{\mathrm{dc}}$ is the bias current and $I_{0}^{\mathrm{ov}}=J W L$ is the critical current of the junction. Perturbation theory based on Eq. (1) (valid for small $\alpha$ and $\eta$ ) gives the following result for the ZFS voltages: ${ }^{6}$

$$
V_{\mathrm{ZFS}, N}=N 2 \pi V_{0} \frac{u_{\infty}}{l},
$$

where the appropriate boundary conditions $\left[\phi_{x}(0, t)=\phi_{x}(l, t)=0\right.$, i.e., zero magnetic field] have been used, and where $u_{\infty}$ is the normalized steady-state fluxon velocity,

$$
u_{\infty}=\left[1+(4 \alpha / \pi \eta)^{2}\right]^{-1 / 2} .
$$

$V_{0}=\hbar \omega_{0} / 2 e$ is the voltage corresponding to the maximum plasma frequency. In general we would expect this perturbative approach, which builds on modifications to the exact analytical solutions to the sine-Gordon equation on an infinitely long line, to describe the behavior of the low-order ZFS in the $I-V$ curve of very long junctions (we note that analytical solutions to the finite line case have been obtained recently ${ }^{7}$ ). For short junctions, analytical extensions of Kulik's small-junction $(l<<1)$ theory for FS (Ref. 8) have been worked out and extended to include ZFS. ${ }^{9}$ These models use an approach based on nonlinear self-coupling of cavity waves to solve Eq. (1) employing various types of Ansätze as solutions. For example, Enpuku et al. ${ }^{9}$ used solutions of the following form:

$$
\phi(x, t)=\omega_{J} t+\sum_{n=1}^{m} \phi_{n}(t) \cos (n \pi x / l),
$$

where $\omega_{J}=(2 e / \hbar) V_{\mathrm{dc}}$. Their computations showed that the ZFS may be described by mixing modes and that more than one solution exists even on the first ZFS (degenera- 


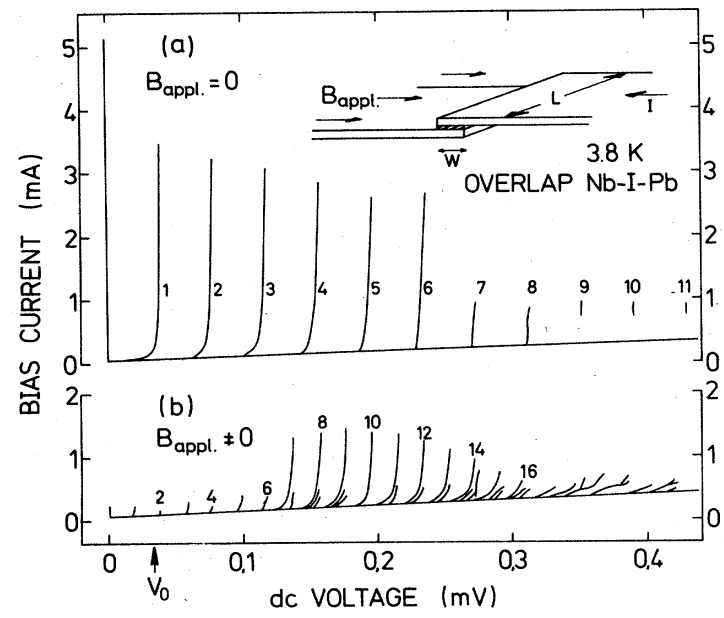

FIG. 1. Overlap junction, $T=3.8 \mathrm{~K}, J=55 \mathrm{~A} / \mathrm{cm}^{2}$, $L \times W=396.0 \times 25.5 \mu \mathrm{m}^{2}, l \approx 6$. The inset shows the junction geometry. (a) $I-V$ curve with 11 zero-field steps $(B=0)$. (b) $I$ $V$ curve with Fiske steps $(B \neq 0)$. We vary $B_{\text {appl }}$ in order to trace out all branches and to find the maximum step heights.

cy). A third approach to the solution of Eq. (1) is direct numerical or analog simulation. For intermediate-length junctions which may be described neither by the perturbative approaches (for long junctions) nor by the multimode self-coupling analyses (for short junctions), direct simulation seems to be necessary. 4,10

\section{EXPERIMENTAL TECHNIQUE}

The experimental radiation data reported here is obtained by detecting the microwave power emitted from one end of the junction. For a mode with $N$ bunched solitons, the frequency of the $M$ th harmonic of the radiation detected when the junction is biased on the Nth ZFS is given by the generalized Josephson relation ${ }^{11}$

$$
v_{\mathrm{det}}=\frac{1}{2} \frac{M}{N} \frac{2 e}{h} V_{\mathrm{dc}} \text {. }
$$

Sample preparation and experimental technique, including the microwave detection system, have been described, in principle, in previous publications. ${ }^{11,12}$ The microstrip coupling scheme was changed somewhat. It consisted of a microstrip antenna at a fixed distance of about $10 \mu \mathrm{m}$ from one end of the junction and a moveable ground plane $(\mathrm{Nb})$ whose distance to the substrate could be tuned from the top of the cryostat (inverted microstripline geometry). The overall noise figure for the $X$-band $\mathrm{mi}$ crowave detection system was about $4.5 \mathrm{~dB}$. The spectral resolution was $1 \mathrm{kHz}$.

The junction used for this study was a $\mathrm{Nb}-\mathrm{Nb}_{x} \mathrm{O}_{y}-\mathrm{Pb}$ overlap junction with $W=25.5 \mu \mathrm{m}$ and $L=396.0 \mu \mathrm{m}$. Viewed in a microscope the overlap was nearly perfect (to within $0.5 \mu \mathrm{m}$ ). The critical current density was about 50 $\mathrm{A} / \mathrm{cm}^{2}$ at $4.2 \mathrm{~K}$, giving $\lambda_{J} \approx 70 \mu \mathrm{m}$ and $l \approx 5$ to 6 . Great care was taken in order to protect the junction from external noise sources. These precautions included a series of microwave rejection filters and isolators, distributed rf filtering of the bias leads and magnetic shielding. For the radiation data reported here an extremely high stability of

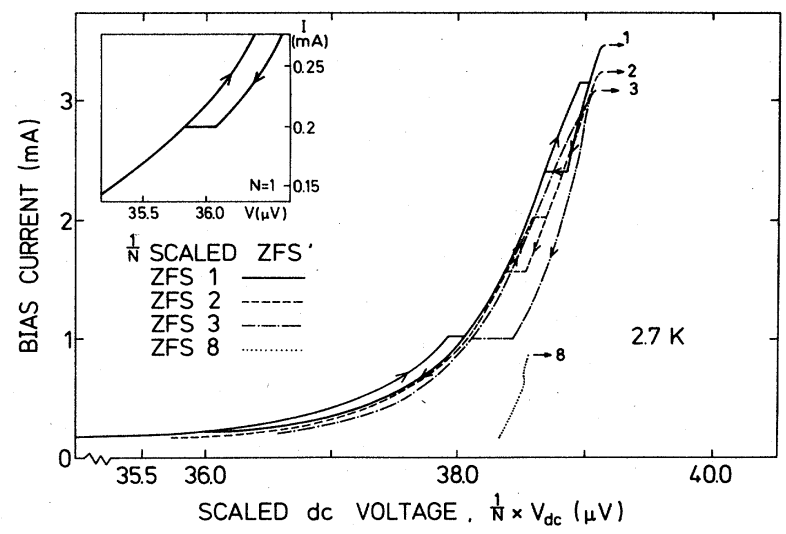

FIG. 2. Same junction as in Fig. $1, T=2.7 \mathrm{~K}$. Measured ZFS Nos. 1, 2, 3, and 8 all scaled by $1 / N$ along the voltage axis. The inset shows the lower part of the hysteresis curve for ZFS 1 with enhanced current sensitivity.

the bias parameters $\left(T, B_{\text {appl }}\right.$, and $\left.I_{\mathrm{dc}}\right)$ was essential. Typical frequency tuning rates $\Delta v / \Delta T, \Delta v / \Delta B$, and $\Delta v / \Delta I$ were $0.1 \mathrm{MHz} / \mathrm{mK}, 0.5 \mathrm{MHz} / \mu \mathrm{T}$, and 2 $\mathrm{MHz} / \mu \mathrm{A}$ (dynamic resistance $\approx 10 \mathrm{~m} \Omega$ ). The electronic temperature stabilization system was capable of maintaining a constant temperature to within $10 \mu \mathrm{K}$. A small external magnetic field could be applied in the plane of the junction (see inset in Fig. 1). When recording the ZFS the strength of this field was set to give maximum critical current $\left(B_{\text {appl }} \approx 5 \mu \mathrm{T}\right) .{ }^{13}$ Experimentally this field also corresponded to an extremum in the frequency of the radiation emitted by the junction biased on one of the ZFS. $^{14}$

\section{EXPERIMENTAL RESULTS}

Figure 1(a) reproduces the $I-V$ curve of the junction at 4.2 $\mathrm{K}$ with the six ordinary ZFS and the five extra, smaller high-order steps $(N=7-11)$ with distorted shapes [see also Fig. 3(c) below]. Up to 21 steps have been observed in a junction of similar normalized length $(l \approx 6) .{ }^{14}$ In Fig. $1(b)$ is shown the $I-V$ curve with $B_{\text {appl }} \neq 0$. This curve was recorded with varying magnitude of the applied field in order to trace out all the Fiske-step branches and the maximum heights of the steps.

With use of a much higher voltage sensitivity than in Fig. 1, Fig. 2 shows the first three plus the eighth ZFS all scaled by $1 / N$ along the voltage axis [see Eq. (2)]. The branching (hysteretic) fine structure on the three lowerorder steps is evident. We note that the magnetic and thermal tuning of the shape of these structures is very strong. Here we shall only deal with structures in zero field. Note the voltage sensitivity which is necessary in order to resolve the close-lying branches on the ZFS ( $\Delta V_{\mathrm{dc}} \approx 100 \mathrm{nV}$ in a $20-\mathrm{Hz}$ bandwidth). On some of the ZFS [e.g., on the fourth step shown in Fig. 3(b) below] the higher-voltage branches of the step could only be accessed by tuning the loss $(\alpha)$ in the junction by varying the temperature. $^{15}$ 
In Figs. 3(a), 3(b), and 3(c) we compare the measured dc $I$ - $V$ curves with the measured $v_{\mathrm{det}}$-versus- $I$ curves for ZFS Nos. 1, 4, and 8 (no scaling of the voltage axis). Using a small test junction on the same substrate as the long junction, we performed an accurate in situ dc voltage calibration on the $5 \times 10^{-4}$ level. This was done by using rfinduced steps in the $I-V$ curve of the small diode obtained by irradiating it with the output from a phase-locked $X$ band microwave source. The voltage measuring circuit could then be calibrated using the Josephson relation, $V_{\mathrm{dc}}=m h v / 2 e$, where $m$ refers to the $m$ th $\mathrm{rf}$-induced step. The limiting factor for the accuracy of this calibration was the temperature drift of the dc voltage amplifier. As may be seen by inspection of Figs. 3(a) and 3(b), for ZFS 1 and 4 the measured frequencies and dc voltages fulfill Eq. (5) to within the experimental uncertainty. Such agreement is representative for the lower-order ZFS ( $N=1-6$ at $4.2 \mathrm{~K}$ ). For the higher-order ZFS, significant deviations from Eq. (5) were observed. Figure 3(c) shows ZFS 8. Here the frequency of the radiation detected from the junction was about $1 \%$ higher than the corresponding dc voltage and the observed relative changes in $v_{\mathrm{det}}$ and $V_{\mathrm{dc}}$ were not correlated. Similar strong deviations from the generalized Josephson relation [Eq. (5)] were observed for the next high-order irregular ZFS. It should be pointed out that for all the ZFS, the measured linewidth of the detected radiation was on the order of $5 \mathrm{kHz}$. A similar observation of "non-Josephson" radiation has been reported by Langenberg et al. ${ }^{16}$ from a long tunnel junction presumably biased on a Fiske step (in a magnetic field).

\section{DISCUSSION}

As shown in Ref. 11, the observation of radiation in the same frequency range on all the ZFS may be explained by assuming $N$ bunched fluxons moving on the Josephson transmission line biased on the Nth ZFS. Branching on some of the regular low-order ZFS [e.g., ZFS No. 4 in Fig. 3(b)] may be explained by assuming different configurations of bunched fluxons. ${ }^{17}$ This picture can account for the branching on the higher-order regular ZFS since the different configurations of fluxons and antifluxons will move with slightly different average velocity. The new observation of branching on ZFS 1 is, however, difficult to explain on the basis of the multiexcitation bunching picture, since this would require an asymmetrical configuration, which logically seems to be incompatible with the symmetric boundary conditions in the absence of a magnetic field.

Likewise, the appearance of the extra, high-order ZFS does not fit into the multiexcitation soliton model. Other modes are evidently excited on the line. We have observed that the extra, high-order ZFS develop into regular, loworder ZFS with decreasing $\lambda_{J}$ (i.e., with decreasing temperature). The distorted shape and the non-Josephson radiation therefore seem linked with the packing of more than $l$ fluxons on the line. We also find the gradual change in the heights and the shape of the FS [Fig. 1(b)] to be suggestive of a gradual transition between modes as we proceed from the low-order to the high-order steps. As far as the non-Josephson radiation is concerned, it has
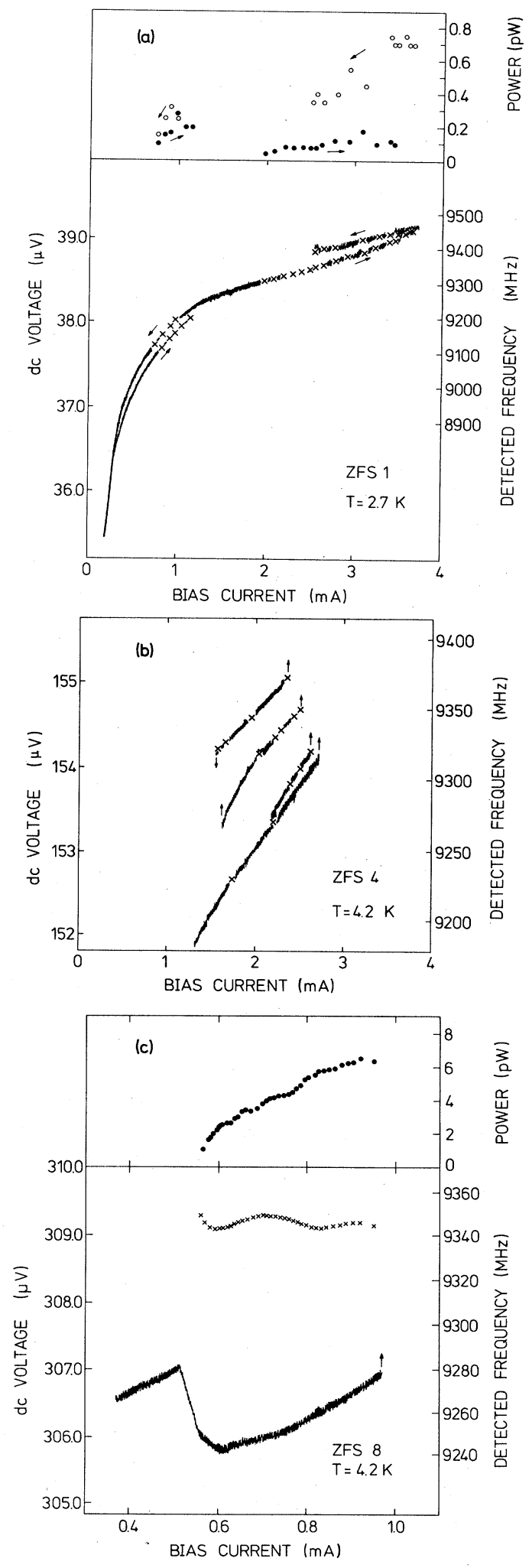

FIG. 3. (a) ZFS 1, (b) ZFS 4, and (c) ZFS 8: Measured dc $V$ $I$ curves (lines) and $v_{\mathrm{det}}-I$ curves ( $\times$ 's). The $V_{\mathrm{dc}}$ and $v_{\mathrm{det}}$ axes are connected by Eq. (5). For ZFS 1 and 8 the detected microwave power as a function of the bias current is also shown, (a) and (c). 
been shown ${ }^{18}$ how a current-biased $(0+1)$-dimensional Josephson junction (e.g., a point contact) coupled to a resonant structure pumps the resonance parametrically, when the junction is biased at a voltage slightly different from the voltage corresponding to the resonance frequency. Power should also be emitted at the proper Josephson frequency, but that radiation may have been too faint to be observed experimentally.

With regard to the observed branching on the low-order ZFS, other observations ${ }^{19}$ suggest a link to the regular, weak fine structure on the very lowest part of these steps. We speculate that either plasmons, large amplitude plasmon-like excitations, or fractional cavity waves are excited by the fluxon during the reflections at the ends of the junction. The observed sensitivity of the branching to a small applied magnetic field indicates the importance of the boundary conditions at the junction ends in determin- ing the nature of the excited modes. Theoretically, a multimode analysis as given by Enpuku et al. ${ }^{9}$ or an equivalent approach built on a similar Ansatz to the solution of Eq. (1) would be fruitful in order to elucidate the precise nature of the modes that couple to the fluxons, and which from our observations appear to become dominant for the high-order ZFS.

\section{ACKNOWLEDGMENTS}

The authors wish to acknowledge I. Rasmussen for the sample fabrication; G. F. Eriksen and T. Huld for valuable assistance; and O. A. Levring, R. D. Parmentier, N. F. Pedersen, M. R. Samuelsen, D. Welner, and C. A. D. Winther for contributions and stimulating discussions. The financial support from the Danish Natural Science Research Council and the Carlsberg Foundation is gratefully acknowledged.
${ }^{1}$ A. C. Scott, F. Y. F. Chu, and S. A. Reible, J. Appl. Phys. 47, 3272 (1976).

${ }^{2}$ A. Matsuda and T. Kawakami, Phys. Rev. Lett. 51, 694 (1983).

${ }^{3}$ T. A. Fulton and R. C. Dynes, Solid State Commun. 12, 57 (1973).

${ }^{4}$ O. H. Olsen and M. R. Samuelsen, J. Appl. Phys. 52, 6247 (1981).

${ }^{5}$ D. W. MacLaughlin and A. C. Scott, Phys. Rev. A 18, 1652 (1978).

${ }^{6}$ O. A. Levring, N. F. Pedersen, and M. R. Samuelsen, Appl. Phys. Lett. 40, 846 (1982).

${ }^{7}$ P. Bodin, N. F. Pedersen, M. R. Samuelsen, and D. Welner, in Proceedings of the Applied Superconductivity Conference, San Diego, 1984 [IEEE Trans. Magn. (to be published)].

${ }^{8}$ I. O. Kulik, Zh. Tekh. Fiz. 37, 157 (1967) [Sov. Phys.-Tech. Phys. 12, 111 (1967)].

${ }^{9}$ K. Takanaka, Solid State Commun. 29, 443 (1979); Y.-S. Gou and C.-S. Chung, J. Low Temp. Phys. 37, 367 (1979); K. Enpuku, K. Yoshida, and F. Irie, J. Appl. Phys. 52, 344 (1981); J.-J. Chang, J. T. Chen, and M. R. Scheuermann, Phys. Rev. B 25, 151 (1982); H. Kawamoto, Prog. Theor. Phys. 70, 1171 (1983).

10P. S. Lomdahl, O. H. Soerensen, and P. L. Christiansen, Phys. Rev. B 25, 5737 (1983); S. N. Erné, A. Ferrigno, and R. D. Parmentier, ibid. 27, 5440 (1983); M. P. Soerensen, R. D. Parmentier, P. L. Christiansen, O. Skovgaard, B. Dueholm, E. Joergensen, V. Koshelets, O. A. Levring, R. Monaco, J. Mygind, N. F. Pedersen, and M. R. Samuelsen, ibid. 30, 2640
(1984).

${ }^{11}$ B. Dueholm, O. A. Levring, J. Mygind, N. F. Pedersen, O. H. Soerensen, and M. Cirillo, Phys. Rev. Lett. 46, 1299 (1981).

${ }^{12}$ E. Joergensen, V. P. Koshelets, R. Monaco, J. Mygind, M. R. Samuelsen, and M. Salerno, Phys. Rev. Lett. 49, 1093 (1982).

${ }^{13}$ J. Bindslev Hansen, G. F. Eriksen, J. Mygind, M. R. Samuelsen, and S. A. Vasenko, in Proceedings of the 17th International Conference on Low Temperature Physics-LT-17, edited by U. Eckern et al. (Elsevier, Amsterdam, 1984), p. 699.

${ }^{14} \mathrm{O}$. A. Levring, M. Sc. thesis, The Technical University of Denmark, 1982 (unpublished).

${ }^{15}$ B. Kofoed, J. Mygind, N. F. Pedersen, M. R. Samuelsen, D. Welner, and C. A. D. Winther, in Proceedings of the 17th International Conference on Low Temperature Physics-LT-17, edited by U. Eckern et al. (Elsevier, Amsterdam, 1984), p. 709.

${ }^{16}$ D. N. Langenberg, W. H. Parker, and B. N. Taylor, Phys. Lett. 22, 259 (1966).

${ }^{17}$ P. S. Lomdahl, O. H. Soerensen, and P. L. Christiansen, Phys. Rev. B 25, 5737 (1983).

${ }^{18}$ A. N. Vystavkin, V. N. Gubankov, L. S. Kuz'min, K. K. Likharev, V. V. Migulin, and V. K. Semenov, Rev. Phys. Appl. 9, 79 (1974); V. N. Gubankov, L. S. Kuz'min, K. K. Likharev, and V. K. Semenov, Zh. Eksp. Teor. Fiz. 68, 230 (1975) [Sov. Phys.-JETP 41, 1150 (1976)].

${ }^{19}$ N. F. Pedersen and D. Welner, Phys. Rev. B 29, 2551 (1984); M. R. Scheuermann, T. V. Rajeevakumar, J.-J. Chang, and J. T. Chen, Physica 107B, 543 (1981). 